TRANSACTIONS OF THE

AMERICAN MATHEMATICAL SOCIETY

Volume 362, Number 4, April 2010, Pages 1681-1698

S 0002-9947(09)05011-9

Article electronically published on November 17, 2009

\title{
THE LI-YAU-HAMILTON INEQUALITY FOR YAMABE FLOW ON A CLOSED CR 3-MANIFOLD
}

\author{
SHU-CHENG CHANG, HUNG-LIN CHIU, AND CHIN-TUNG WU
}

\begin{abstract}
We deform the contact form by the (normalized) CR Yamabe flow on a closed spherical CR 3-manifold. We show that if a contact form evolves with positive Tanaka-Webster curvature and vanishing torsion from initial data, then we obtain a new Li-Yau-Hamilton inequality for the CR Yamabe flow. By combining this parabolic subgradient estimate with a compactness theorem of a sequence of contact forms, it follows that the CR Yamabe flow exists for all time and converges smoothly to, up to the CR automorphism, a unique limit contact form of positive constant Webster scalar curvature on a closed CR 3-manifold, which is CR equivalent to the standard CR 3-sphere with positive Tanaka-Webster curvature and vanishing torsion.
\end{abstract}

\section{INTRODUCTION}

Let $\left(M^{2 n+1}, J\right)$ denote a closed $(2 n+1)$-dimensional $C R$ manifold (we refer to section 2 for the details). The CR Yamabe problem is to find a contact form on $M$ with constant Tanaka-Webster curvature. In serial papers ([JL1, JL2, JJL3]), D. Jerison and J. Lee initiated the study of this problem. They confirmed the CR Yamabe problem in the case where the CR Yamabe constant $\lambda(M)$ is less than the one $\lambda\left(\mathbf{S}^{2 n+1}\right)$ for a standard CR sphere $\left(\mathbf{S}^{2 n+1}, \widehat{J}\right)$. Their methods can be compared to the partial completion of the proof of the Riemannian Yamabe problem by $\mathrm{T}$. Aubin $(\underline{\underline{A}})$. By the pseudohermitian analogue, the remaining cases should be solved using a $\mathrm{CR}$ version of the positive pseudohermitian-mass conjecture which is not available at this present stage of research.

In this paper, let $\left(M, J, \theta_{0}\right)$ denote a closed pseudohermitian 3-manifold and $\theta_{(t)}$ be a family of contact forms on $M$. The (normalized) CR Yamabe flow is evolved in the contact form as follows:

$$
\partial_{t} \theta_{(t)}=2\left(r_{(t)}-W_{(t)}\right) \theta_{(t)},
$$

where $W_{(t)}$ is the Tanaka-Webster curvature of $\theta_{(t)}$ and $r_{(t)}$ is the mean value of $W_{(t)}$, i.e., $r_{(t)}=\int_{M} W_{(t)} d \mu_{t} / \int_{M} d \mu_{t}, d \mu_{t}=\theta_{(t)} \wedge d \theta_{(t)}$ is the volume form of $\theta_{(t)}$.

Received by the editors January 23, 2007.

2000 Mathematics Subject Classification. Primary 32V20; Secondary 53C44.

Key words and phrases. Li-Yau-Hamilton inequality, CR Bochner formula, Tanaka-Webster curvature, pseudoharmonic manifold, CR pluriharmonic operator, CR Paneitz operator, subLaplacian, subgradient estimate, CR Yamabe flow, positive mass theorem.

This research was supported in part by the NSC of Taiwan. 
We write $\theta_{(t)}=e^{2 \lambda_{(t)}} \theta_{0}$ with respect to a fixed contact form $\theta_{0}$. Then we can express the equation (1.1) in terms of $\lambda_{(t)}$ by

$$
\left\{\begin{array}{l}
\frac{\partial \lambda_{(t)}}{\partial t}=r_{(t)}-W_{(t)}, \\
\theta_{(t)}=e^{2 \lambda_{(t)} \theta_{0},} \\
\lambda(p, 0)=\lambda_{0}(p) .
\end{array}\right.
$$

The corresponding Yamabe flow on a Riemannian manifold $\left(N^{m}, u_{(t)}^{\frac{4}{m-2}} g_{0}\right)$ can be read as

$$
\left\{\begin{array}{l}
\frac{\partial u_{(t)}}{\partial t}=\left(r_{(t)}-S_{(t)}\right) u, \\
g_{(t)}=u_{(t)}^{\frac{4}{m-2}} g_{0}, \quad u_{(t)}>0, \\
u(p, 0)=u_{0}(p),
\end{array}\right.
$$

where $S_{(t)}$ is the scalar curvature of $g_{(t)}$ and $r_{(t)}$ is the mean value of $S_{(t)}$.

B. Chow $([\mathrm{C}]$ ) obtained the Harnack inequality for the solution of the Riemannian Yamabe flow and proved that the asymptotic convergence of solutions of the flow provided that $\left(M, g_{0}\right)$ is locally conformally flat and $g_{0}$ has positive Ricci curvature. By using Schoen-Yau's positive mass theorem ([SY]), the second assumption was removed by R. Ye $([\mathrm{Y}]$ ). He showed that the flow converges to a metric of positive constant scalar curvature whenever $\left(M, g_{0}\right)$ is locally conformally flat. H. Schwetlick and M. Struwe obtained similar results with the help of the concentration compactness theorem ([SS]).

We observe that, by using Hamilton's general method, J.-H. Cheng and the first named author ([CC]) obtained the standard Harnack inequality for the CR Yamabe flow (1.1) on a closed spherical CR 3-manifold with positive Tanaka-Webster curvature and vanishing torsion.

In this paper, by using the method of $\mathrm{Li}$ and Yau $([\mathrm{LY}])$, we get a new LiYau-Hamilton inequality for the flow (1.1) on a closed spherical CR 3-manifold with positive Tanaka-Webster curvature and vanishing torsion. With the help of a compactness theorem of a sequence of contact forms, we prove the asymptotic convergence of solutions of the CR Yamabe flow (1.1) as $t \rightarrow \infty$.

We first recall some previous results $([\mathrm{CCW}])$. Let $(M, J, \bar{\theta})$ denote a closed pseudohermitian 3-manifold, and we consider a positive solution $u(x, t)$ of the CR heat equation with respect to the sub-Laplacian $\bar{\Delta}_{b}$

$$
\left(\bar{\Delta}_{b}-\frac{\partial}{\partial t}\right) u(x, t)=0
$$

on $M \times[0, \infty)$. By using the arguments of $[\mathrm{LY}]$, we are able to derive the CR version of the parabolic Li-Yau gradient estimate and the Li-Yau inequality for a positive solution $u(x, t)$ of (1.4) on $M \times[0, \infty)$ with the pluriharmonic function as initial data.

Proposition 1.1 ([CCW] $)$. Let $(M, J, \bar{\theta})$ be a closed pseudohermitian 3-manifold of vanishing torsion and nonnegative Tanaka-Webster curvature. If $u(x, t)$ is a positive solution of the $C R$ heat equation (1.4) on $M \times[0, \infty)$ with $P_{0} u(x, 0)=0$, then $u$ satisfies the estimate

$$
\frac{\left|\bar{\nabla}_{b} u\right|^{2}}{3 u^{2}}+\frac{u_{t}}{u} \leq \frac{3}{t}
$$

on $M \times(0, \infty)$. 
Integrating (1.5) from time $t_{1}$ to time $t_{2}$, we obtain the following $\mathrm{CR}$ version of the Li-Yau inequality.

Corollary 1.2 ([CCW $]$ ). Let $(M, J, \bar{\theta})$ be a closed pseudohermitian 3-manifold of vanishing torsion and nonnegative Tanaka-Webster curvature. If $u(x, t)$ is a positive solution of the $C R$ heat equation (1.4) on $M \times[0, \infty)$ with $P_{0} u(x, 0)=0$, then for any $x_{1}, x_{2}$ in $M$ and $0<t_{1}<t_{2}<\infty$, we have the inequality

$$
u\left(x_{2}, t_{2}\right) \leq u\left(x_{1}, t_{1}\right)\left(\frac{t_{2}}{t_{1}}\right)^{3} \exp \left(\frac{3}{4} L\right)
$$

where

$$
L=\inf _{\gamma} \int_{t_{1}}^{t_{2}}|\dot{\gamma}|^{2} d t
$$

and the infimum is taken over all horizontal curves $\gamma$ with $\gamma\left(t_{1}\right)=x_{1}$ and $\gamma\left(t_{2}\right)=$ $x_{2}$.

Remark 1.3. Due to the CR version of Bochner formula (3.5) involving a term which is the so-called CR Paneitz operator $P_{0}$ and has no analogue in the Riemannian case, the CR version of the parabolic Li-Yau gradient estimate and the Li-Yau inequality are somewhat different from the one of the Riemannian case, as in the remarkable paper of $\mathrm{Li}$ and Yau $([\mathrm{LY}])$. It is also interesting to compare the results of Cao and Yau ([CY]) with ours.

For later use, we call a CR structure $J$ spherical if a Cartan curvature tensor $Q_{11}$ vanishes identically. Here

$$
Q_{11}=\frac{1}{6} W_{11}+\frac{i}{2} W A_{11}-A_{11,0}-\frac{2 i}{3} A_{11, \overline{1} 1} .
$$

Note that $(M, J)$ is called a closed spherical pseudohermitian 3-manifold if $J$ is a spherical structure. We observe that the spherical structure is CR invariant and that a closed spherical pseudohermitian 3-manifold $(M, J)$ is locally CR equivalent to $\left(\mathbf{S}^{3}, \widehat{J}\right)$.

We denote $f_{t}$ by partial differentiation of a smooth function $f$ with respect to the variable $t$ and write $W=W_{(t)}, r=r_{(t)}$, etc., for short in the notation. Now by using the method of Li and Yau $([\mathrm{LY}]$ ) and ([CW $]$, we are able to get the CR version of the parabolic Li-Yau-Hamilton gradient estimate.

Theorem 1.4. Let $\left(M, J, \theta_{(0)}\right)$ be a closed spherical pseudohermitian 3-manifold with vanishing torsion and positive Tanaka-Webster curvature. Then under the Yamabe flow (1.1), the Tanaka-Webster curvature $W$ satisfies the estimate

$$
\frac{4}{3} \frac{\left|\nabla_{b} W\right|^{2}}{W^{2}}+\frac{W_{t}}{W} \leq 6 r+\frac{3}{t}
$$

on $M \times(0, \infty)$.

By integrating (1.7) from time $t_{1}$ to time $t_{2}$, we obtain the following Li-YauHamilton Harnack inequality.

Corollary 1.5. Consider the Yamabe flow under the same assumptions as in Theorem 1.4. Then for all points $x_{1}, x_{2}$ in $M$ and times $0<t_{1}<t_{2} \leq \infty$, we have the inequality

$$
W\left(x_{2}, t_{2}\right) \leq W\left(x_{1}, t_{1}\right)\left(\frac{t_{2}}{t_{1}}\right)^{3} \exp \left(6 R+\frac{3}{16} L\right)
$$


where

$$
R=\int_{t_{1}}^{t_{2}} r_{(t)} d t, L=\inf _{\gamma} \int_{t_{1}}^{t_{2}}|\dot{\gamma}|_{J, \theta_{(t)}}^{2} d t
$$

and the infimum is taken over all Legendrian paths $\gamma$ with $\gamma\left(t_{1}\right)=x_{1}$ and $\gamma\left(t_{2}\right)=$ $x_{2}$.

We have an opposite sign of the term $\frac{W_{t}}{W}$ in (1.7) with the one in Hamilton's paper $([\underline{\mathrm{H}})$ for the Ricci flow due to the CR version of the Bochner formula. Indeed, as in (3.7), one needs to deal with an extra term $\left\langle P W+\bar{P} W, d_{b} W\right\rangle_{L_{\theta}^{*}}$ which is an operator that characterizes CR-pluriharmonic functions in Lemma 3.8. However we are able to control it for a closed spherical pseudohermitian manifold with positive Tanaka-Webster curvature and vanishing torsion. It follows that the $\mathrm{CR}$ version of Li-Yau-Hamilton's inequality (1.7) holds, and then we have the uniformly $L^{p_{-}}$ bound estimate of $W$ as in Lemma 5.2, for $p>2$. As a consequence of (1.7) and a compactness theorem of a sequence of contact forms, we show the convergence of solutions of the CR Yamabe flow (1.1) as follows:

Theorem 1.6. Let $\left(M, J, \theta_{(0)}\right)$ be a closed pseudohermitian 3-manifold which is $C R$ equivalent to $\left(\mathbf{S}^{3}, \widehat{J}\right)$ with vanishing torsion and positive Tanaka-Webster curvature. Then solutions of the CR Yamabe flow (1.1) converge smoothly to, up to the CR automorphism, a unique limit contact form of constant Webster scalar curvature as $t \rightarrow \infty$.

Due to Conjecture 4.8 in section 4, we make a further conjecture:

Conjecture 1.7. Let $\left(M, J, \theta_{(0)}\right)$ be a closed spherical pseudohermitian 3-manifold with vanishing torsion and positive CR Yamabe constant. Then solutions of the $C R$ Yamabe flow (1.1) converge smoothly to, up to the CR automorphism, a unique limit contact form of constant Webster scalar curvature as $t \rightarrow \infty$. As a consequence, there is a contact form of positive constant Tanaka-Webster curvature on such a pseudohermitian 3-manifold.

Besides T. Aubin's ( $\underline{\underline{A}}]$ ) and R. Schoen's ( $\underline{\text { Sch }}$ ) proof, a very different solution to the Riemannian Yamabe problem was introduced by A. Bahri and H. Brezis and can be generalized to the CR Yamabe problem by N. Gamara and Y. Yacoub $(\mathrm{GY}]$ ).

We briefly describe the methods used in our proofs. In section 3, we first derive some basic properties for the CR Yamabe flow. Second, by using the arguments of [LY] and [CCW], we are able to have Li-Yau-Hamilton's inequality for the CR Yamabe flow, and then the CR version of the parabolic gradient estimate is derived on a pseudohermitian 3-manifold. In section 4, by applying the second named author's previous method (Chi1), we are able to get a pseudohermitian compactness theorem of a sequence of contact forms. In section 5, we first derive the uniformly $L^{p}$-bound estimate of $W$ from the subgradient estimate in Theorem 1.4. By combining a compactness theorem of a sequence of contact forms in section 4 , we are able to get the uniformly $S^{2, p}$-estimates for any fixed $p>2$ and then the asymptotic convergence of solutions of the CR Yamabe flow (1.1).

\section{Preliminaries}

Let us first review some basic material in pseudohermitian geometry ([T], $[\mathrm{W}]$ ). Let $M$ be a closed 3-manifold with an oriented contact structure $\xi$. There always 
exists a global contact form $\theta$, obtained by patching together local ones with a partition of unity. The characteristic vector field of $\theta$ is the unique vector field $T$ such that $\theta(T)=1$ and $d \theta(T, \cdot)=0$. A $C R$-structure $J$ compatible with $\xi$ is a smooth endomorphism $J: \xi \rightarrow \xi$ such that $J^{2}=-i d e n t i t y$. A pseudohermitian structure compatible with $\xi$ is a $C R$-structure $J$ compatible with $\xi$ together with a global contact form $\theta$.

Given a pseudohermitian structure $(J, \theta)$, we can choose a complex vector field $Z_{1}$, an eigenvector of $J$ with eigenvalue $i$, and a complex 1 -form $\theta^{1}$ such that $\left\{\theta, \theta^{1}, \theta^{\overline{1}}\right\}$ is dual to $\left\{T, Z_{1}, Z_{\overline{1}}\right\}$. It follows that $d \theta=i h_{1 \overline{1}} \theta^{1} \wedge \theta^{\overline{1}}$ for some nonzero real function $h_{1 \overline{1}}$. If $h_{1 \overline{1}}$ is positive, we call such a pseudohermitian structure $(J, \theta)$ positive, and we can choose a $Z_{1}$ (hence $\theta^{1}$ ) such that $h_{1 \overline{1}}=1$. That is to say,

$$
d \theta=i \theta^{1} \wedge \theta^{\overline{1}}
$$

Throughout the paper we'll always assume our pseudohermitian structure $(J, \theta)$ is positive and $h_{1 \overline{1}}=1$. The pseudohermitian connection of $(J, \theta)$ is the connection $\nabla$ on $T M \otimes C$ (and extended to tensors) given by

$$
\nabla Z_{1}=\omega_{1}^{1} \otimes Z_{1}, \quad \nabla Z_{\overline{1}}=\omega_{\overline{1}}^{\overline{1}} \otimes Z_{\overline{1}}, \quad \nabla T=0
$$

in which the 1 -form $\omega_{1}{ }^{1}$ is uniquely determined by the following equation with a normalization condition:

$$
\begin{gathered}
d \theta^{1}=\theta^{1} \wedge \omega_{1}{ }^{1}+A^{1}{ }_{\overline{1}} \theta \wedge \theta^{\overline{1}}, \\
\omega_{1}{ }^{1}+\omega_{\overline{1}}{ }^{\overline{1}}=0 .
\end{gathered}
$$

The coefficient $A^{1} \overline{1}_{\overline{1}}$ is called the (pseudohermitian) torsion. Since $h_{1 \overline{1}}=1$, $A_{\overline{1} \overline{1}}=h_{1 \overline{1}} A^{1} \overline{1}_{1}=A^{1}{ }_{\overline{1}}$, and $A_{11}$ is just the complex conjugate of $A_{\overline{1} \overline{1}}$. Differentiating $\omega_{1}^{1}$ gives

$$
d \omega_{1}^{1}=W \theta^{1} \wedge \theta^{\overline{1}}+2 i \operatorname{Im}\left(A_{11, \overline{1}} \theta^{1} \wedge \theta\right),
$$

where $W$ is the Tanaka-Webster curvature.

We can define the covariant differentiations with respect to the pseudohermitian connection. For instance, $f_{1}=Z_{1} f, f_{1 \overline{1}}=Z_{\overline{1}} Z_{1} f-\omega_{1}{ }^{1}\left(Z_{\overline{1}}\right) Z_{1} f$, and $f_{0}=T f$ for a function $f$. We define the subgradient operator $\nabla_{b}$ and the sublaplacian operator $\Delta_{b}$ by $\nabla_{b} f=f_{\overline{1}} Z_{1}+f_{1} Z_{\overline{1}}$ and $\Delta_{b} f=f_{1 \overline{1}}+f_{\overline{1} 1}$, respectively. We also define the Levi form $\langle,\rangle_{J, \theta}$ by

$$
\langle V, U\rangle_{J, \theta}=2 d \theta(V, J U)=v_{1} u_{\overline{1}}+v_{\overline{1}} u_{1}
$$

for $V=v_{1} Z_{\overline{1}}+v_{\overline{1}} Z_{1}, U=u_{1} Z_{\overline{1}}+u_{\overline{1}} Z_{1}$ in $\xi$. The associated norm is defined as usual: $|V|_{J, \theta}^{2}=\langle V, V\rangle_{J, \theta}$. Next we recall below the Folland-Stein space $S^{k, p}$.

Define

$$
(V, U)_{J, \theta}=\int_{M}\langle V, U\rangle_{J, \theta} \theta \wedge d \theta .
$$

For a vector $X \in \xi$, we define $|X|^{2} \equiv\langle X, X\rangle_{J, \theta}$. It follows that $\left|\nabla_{b} f\right|^{2}=2 f_{1} f_{\overline{1}}$ for a real valued smooth function $f$. Also, the square modulus of the sub-Hessian $\nabla_{b}^{2} f$ of $f$ reads $\left|\nabla_{b}^{2} f\right|^{2}=2 f_{11} f_{\overline{1} \overline{1}}+2 f_{1 \overline{1}} f_{\overline{1} 1}$. Let $D$ denote a differential operator acting on functions. We say $D$ has weight $m$, denoted $w(D)=m$, if $m$ is the smallest integer such that $D$ can be locally expressed as a polynomial of degree $m$ in vector fields tangent to the contact bundle $\xi$. We define the Folland-Stein space $S^{k, p}$ of functions on $M$ by

$$
S^{k, p}=\left\{f \in L^{p}: D f \in L^{p} \text { whenever } w(D) \leq k\right\} .
$$


We define the $L^{p}$ norm of $\nabla_{b} f, \nabla_{b}^{2} f, \ldots$ to be $\left(\int\left|\nabla_{b} f\right|^{p} \theta \wedge d \theta\right)^{1 / p},\left(\int\left|\nabla_{b}^{2} f\right|^{p} \theta \wedge d \theta\right)^{1 / p}$, $\ldots$, respectively, as usual. So it is natural to define the $S^{k, p}$ norm of $f \in S^{k, p}$ as follows:

$$
\|f\|_{S^{k, p}}=\left(\sum_{0 \leq j \leq k}\left\|\nabla_{b}^{j} f\right\|_{L^{p}}^{p}\right)^{1 / p} .
$$

The function space $S^{k, p}$ with the above norm is a Banach space for $k \geq 0,1<p<$ $\infty$. There are also embedding theorems of Sobolev type. For instance, $S^{2,2} \subset S^{1,4}$ (for $\operatorname{dim} M=3$ ). We refer the reader to [FS] and [Fo for more discussions on these spaces. For all $Z=x^{1} Z_{1} \in T_{1,0}$, one defines

$$
\begin{aligned}
& \operatorname{Ric}(Z, Z)=W x^{1} x^{\overline{1}}, \\
& \operatorname{Tor}(Z, Z)=2 \operatorname{Re} i A_{\overline{1} \overline{1}} x^{\overline{1}} x^{\overline{1}} .
\end{aligned}
$$

A piecewise smooth curve $\gamma:[0,1] \rightarrow M$ is said to be horizontal if $\gamma^{\prime}(t) \in \xi$ whenever $\gamma^{\prime}(t)$ exists. The length of $\gamma$ is then defined by

$$
l(\gamma)=\int_{0}^{1}\left|\gamma^{\prime}(t)\right|_{J, \theta} d t
$$

The Carnot-Carathéodory distance between two points $p, q \in M$ is defined by

$$
d(p, q)=\inf \left\{l(\gamma) \mid \gamma \in C_{p, q}\right\},
$$

where $C_{p, q}$ is the set of all horizontal curves which join $p$ and $q$. By the Chow connectivity theorem [Cho, there always exists a horizontal curve joining $p$ and $q$, so the distance is finite.

\section{The Subgradient estimate and Li-Yau-Hamilton InEQUAlity}

In this section, we first derive some basic facts under the CR Yamabe flow (1.1). Then, by using the arguments of [LY], we are able to derive an estimate on the derivative of the Tanaka-Webster curvature $W$ on a pseudohermitian 3-manifold. This is the so-called the Li-Yau-Hamilton inequality for the CR Yamabe flow.

Lemma 3.1. Under the Yamabe flow (1.1), we have

$$
\begin{aligned}
W_{t} & =4 \Delta_{b} W+2 W(W-r), \\
\partial_{t}\left(A_{11}\right) & =2(W-r) A_{11}-2 i W_{11},
\end{aligned}
$$

where $\Delta_{b}, A_{11}$, and covariant derivatives are with respect to $\theta_{(t)}$.

By applying the maximum principle to (3.1), we obtain

Lemma 3.2. Suppose $\left(M, J, \theta_{(0)}\right)$ is closed with $W_{(0)}>0$. Then the inequality $W>0$ is preserved under the Yamabe flow (1.1).

Following the same proof of Lemma $3.1 \mathrm{in}[\mathrm{CC}$ to equation (3.2), we have that the torsion vanishes for all time if our CR structure $J$ is spherical.

Lemma 3.3. Suppose $J$ is spherical and $A_{11}=0$ for an initial $\theta_{(0)}$. Then, under the Yamabe flow (1.1), $A_{11}$ vanishes for all $\theta_{(t)}$. 
Since the volume of $M$ is preserved along the Yamabe flow (1.1), and by using the equation (3.1) of $W$, we get

Lemma 3.4. Under the Yamabe flow (1.1), we have

$$
\frac{d}{d t} r=-2 \int_{M}(W-r)^{2} d \mu / \int_{M} d \mu .
$$

This implies that $r_{(t)}$ is decreasing along the Yamabe flow (1.1).

The following formulae will be used as we compute the evolution equations of $\Delta_{b} W$ and $\left|\nabla_{b} W\right|^{2}$.

Lemma 3.5. Under the Yamabe flow (1.1), we have

$$
\begin{aligned}
\partial_{t}\left(\Delta_{b} f\right) & =\Delta_{b}\left(f_{t}\right)+2(W-r) \Delta_{b} f-2\left\langle\nabla_{b} W, \nabla_{b} f\right\rangle_{L_{\theta}}, \\
\partial_{t}\left(\left|\nabla_{b} f\right|^{2}\right) & =2(W-r)\left|\nabla_{b} f\right|^{2}+2\left\langle\nabla_{b} f, \nabla_{b} f_{t}\right\rangle_{L_{\theta}}
\end{aligned}
$$

for a (smooth) real-valued function $f=f(x, t)$ defined on $M \times R$.

Let $W$ be a positive solution of equation (3.1) and let $\varphi=\log W$; then $\varphi$ satisfies the equation

$$
\left(\partial_{t} / 4-\Delta_{b}\right) \varphi=\left|\nabla_{b} \varphi\right|^{2}+\frac{1}{2}(W-r) .
$$

We first recall the following formulae that we need in the proof of the subgradient estimate. The first is the CR version Bochner formula involving the CR Paneitz operator.

We define

$$
P \varphi=\left(\varphi_{1}{ }_{1}{ }_{1}+i A_{11} \varphi^{1}\right) \theta^{1}=P \varphi=\left(P_{1} \varphi\right) \theta^{1},
$$

which is an operator that characterizes CR-pluriharmonic functions ([L2]). Here $P_{1} \varphi=\varphi_{\overline{1}}{ }^{\overline{1}}{ }_{1}+i A_{11} \varphi^{1}$ and $\bar{P} \varphi=\left(\bar{P}_{1}\right) \theta^{\overline{1}}$, the conjugate of $P$. The CR Paneitz operator $P_{0}$ is defined by

$$
P_{0} \varphi=4\left(\delta_{b}(P \varphi)+\bar{\delta}_{b}(\bar{P} \varphi)\right),
$$

where $\delta_{b}$ is the divergence operator that takes $(1,0)$-forms to functions by $\delta_{b}\left(\sigma_{1} \theta^{1}\right)=$ $\sigma_{1}{ }^{1}$, and similarly, $\bar{\delta}_{b}\left(\sigma_{\overline{1}} \theta^{\overline{1}}\right)=\sigma_{\overline{1}}{ }^{\overline{1}}$. We observe that

$$
\int\left\langle P \varphi+\bar{P} \varphi, d_{b} \varphi\right\rangle_{L_{\theta}^{*}} d \mu=-\frac{1}{4} \int P_{0} \varphi \cdot \varphi d \mu .
$$

Lemma 3.6 ([Chi2], [CCC]). For a real function $\varphi$,

$$
\begin{aligned}
\frac{1}{2} \Delta_{b}\left|\nabla_{b} \varphi\right|^{2}= & \left|\left(\nabla^{H}\right)^{2} \varphi\right|^{2}+3\left\langle\nabla_{b} \varphi, \nabla_{b} \Delta_{b} \varphi\right\rangle_{L_{\theta}} \\
& +(2 \operatorname{Ric}-3 \text { Tor })\left(\left(\nabla_{b} \varphi\right)_{C},\left(\nabla_{b} \varphi\right)_{C}\right) \\
& -4\left\langle P \varphi+\bar{P} \varphi, d_{b} \varphi\right\rangle_{L_{\theta}^{*}},
\end{aligned}
$$

where $\left(\nabla_{b} \varphi\right)_{\mathbf{C}}=\varphi_{\overline{1}} Z_{1}$ is the corresponding complex (1,0)-vector field of $\nabla_{b} \varphi$ and $d_{b} \varphi=\varphi_{1} \theta^{1}+\varphi_{\overline{1}} \theta^{\overline{1}}$.

Lemma 3.7. Let $\varphi=\log W$, for a positive smooth function $W$. Then

$$
\begin{aligned}
& \left\langle P \varphi+\bar{P} \varphi, d_{b} \varphi\right\rangle_{L_{\theta}^{*}} \\
& =W^{-2}\left\langle P W+\bar{P} W, d_{b} W\right\rangle_{L_{\theta}^{*}}-\frac{1}{2}\left\langle\nabla_{b} \varphi, \nabla_{b}\left|\nabla_{b} \varphi\right|^{2}\right\rangle-\frac{1}{2} W^{-1} \Delta_{b} W\left|\nabla_{b} \varphi\right|^{2} .
\end{aligned}
$$


Proof. Let $Q(x)=\left|\nabla_{b} \varphi\right|^{2}(x)$. We compute

$$
\begin{aligned}
\nabla_{b} Q & =Q_{\overline{1}} Z_{1}+Q_{1} Z_{\overline{1}} \\
& =2 \nabla_{b}\left(\varphi_{1} \varphi_{\overline{1}}\right) \\
& =2\left(\frac{W^{2} W_{1} W_{\Pi 1}+W^{2} W_{\bar{T}} W_{1 \top}-2 W W_{T}^{2} W_{1}}{W^{4}}\right) Z_{1}+\text { complex conjugate. }
\end{aligned}
$$

It follows that

$$
\begin{aligned}
P_{1} \varphi & =\varphi_{\overline{1} 11}+i A_{11} \varphi_{\overline{1}} \\
& =\frac{W^{3} W_{\overline{1} 11}-W^{2} W_{\overline{1}} W_{11}-2 W^{2} W_{1} W_{\overline{1} 1}+2 W W_{1}^{2} W_{\overline{1}}}{W^{4}}+i A_{11} \frac{W_{\overline{1}}}{W} \\
& =\frac{P_{1} W}{W}-\frac{1}{2} Q_{1}-\frac{W_{1} W_{\overline{1} 1}}{W^{2}} \\
& =\frac{P_{1} W}{W}-\frac{1}{2} Q_{1}-\varphi_{1} \frac{W_{\overline{1} 1}}{W} .
\end{aligned}
$$

Thus

$$
\begin{aligned}
4\left\langle P \varphi+\bar{P} \varphi, d_{b} \varphi\right\rangle_{L_{\theta}^{*}} & =4\left\langle\left(P_{1} \varphi\right) \theta^{1}+\left(\bar{P}_{1} \varphi\right) \theta^{\overline{1}}, \varphi_{1} \theta^{1}+\varphi_{\overline{1}} \theta^{\overline{1}}\right\rangle_{L_{\theta}^{*}} \\
= & 4\left[\left(P_{1} \varphi\right) \varphi_{\overline{1}}+\left(\bar{P}_{1} \varphi\right) \varphi_{1}\right] \\
= & 4\left(\frac{P_{1} W}{W}-\frac{1}{2} Q_{1}-\varphi_{1} \frac{W_{\overline{1} 1}}{W}\right) \varphi_{\overline{1}}+\text { complex conjugate } \\
= & 4 \frac{\left\langle P W+\bar{P} W, d_{b} W\right\rangle_{L_{\theta}^{*}}}{W^{2}}-2\left\langle\nabla_{b} \varphi, \nabla_{b}\left|\nabla_{b} \varphi\right|^{2}\right\rangle \\
& -2\left(\frac{\Delta_{b} W}{W}\left|\nabla_{b} \varphi\right|^{2}\right) .
\end{aligned}
$$

This implies the lemma.

Now we prove the following lemma which is essential in the derivation of our subgradient estimate for the Tanaka-Webster curvature.

Lemma 3.8. Let $\left(M, J, \theta_{(0)}\right)$ be a closed spherical 3-dimensional pseudohermitian manifold with positive Tanaka-Webster curvature and vanishing torsion. Then, under the Yamabe flow (1.1), the function

$$
F=t\left[\left|\nabla_{b} \varphi\right|^{2}+\frac{3}{4} \varphi_{t}-12(W-r)\right]
$$

satisfies the inequality

$$
\begin{aligned}
\left(\Delta_{b}-\partial_{t} / 4\right) F \geq & -\frac{2}{3}\left\langle\nabla_{b} \varphi, \nabla_{b} F\right\rangle+\frac{1}{9 t} F\left[F+\left(12 W-\frac{33}{2} r\right) t-\frac{9}{4}\right] \\
& +\frac{16}{9} t\left|\nabla_{b} \varphi\right|^{4}-7 t W\left|\nabla_{b} \varphi\right|^{2}+7 t(W-r)^{2} \\
& -\frac{21}{8} t r_{t}-8 t W^{-2}\left\langle P W+\bar{P} W, d_{b} W\right\rangle_{L_{\theta}^{*}} .
\end{aligned}
$$


Proof. First differentiating (3.6) in the variable $t$ and by Lemma 3.5, we have

$$
\begin{aligned}
F_{t}= & \frac{1}{t} F+t\left[\left|\nabla_{b} \varphi\right|^{2}+\frac{3}{4} \varphi_{t}-12(W-r)\right]_{t} \\
= & \frac{1}{t} F+t\left[4\left|\nabla_{b} \varphi\right|^{2}+3 \Delta_{b} \varphi-\frac{21}{2}(W-r)\right]_{t} \\
= & \frac{1}{t} F+t\left[8(W-r)\left|\nabla_{b} \varphi\right|^{2}+8\left\langle\nabla_{b} \varphi, \nabla_{b} \varphi_{t}\right\rangle+3 \Delta_{b} \varphi_{t}\right. \\
& \left.+6(W-r) \Delta_{b} \varphi-6\left\langle\nabla_{b} W, \nabla_{b} \varphi\right\rangle-\frac{21}{2}\left(W_{t}-r_{t}\right)\right] \\
= & \frac{1}{t} F+t\left[8\left\langle\nabla_{b} \varphi, \nabla_{b} \varphi_{t}\right\rangle+3 \Delta_{b} \varphi_{t}-6(6 W+r) \Delta_{b} \varphi\right. \\
& \left.-8(5 W+r)\left|\nabla_{b} \varphi\right|^{2}-21 W(W-r)+\frac{21}{2} r_{t}\right] .
\end{aligned}
$$

By the Bochner formula (3.5) to equation (3.6) and Lemma 3.7 we obtain

$$
\begin{aligned}
\Delta_{b} F= & t\left[\Delta_{b}\left|\nabla_{b} \varphi\right|^{2}+\frac{3}{4} \Delta_{b} \varphi_{t}-12 \Delta_{b}(W-r)\right] \\
= & t\left[2\left|\left(\nabla^{H}\right)^{2} \varphi\right|^{2}+6\left\langle\nabla_{b} \varphi, \nabla_{b} \Delta_{b} \varphi\right\rangle\right. \\
& +2(2 R i c-3 T o r)\left(\left(\nabla_{b} \varphi\right)_{C},\left(\nabla_{b} \varphi\right)_{C}\right) \\
& \left.-8\left\langle P \varphi+\bar{P} \varphi, d_{b} \varphi\right\rangle_{L_{\theta}^{*}}+\frac{3}{4} \Delta_{b} \varphi_{t}-12 \Delta_{b} W\right] \\
\geq \quad & t\left[4\left|\varphi_{11}\right|^{2}+\left(\Delta_{b} \varphi\right)^{2}+6\left\langle\nabla_{b} \varphi, \nabla_{b} \Delta_{b} \varphi\right\rangle+2 W\left|\nabla_{b} \varphi\right|^{2}\right. \\
& \left.-8\left\langle P \varphi+\bar{P} \varphi, d_{b} \varphi\right\rangle_{L_{\theta}^{*}}+\frac{3}{4} \Delta_{b} \varphi_{t}-12 \Delta_{b} W\right] \\
= & t\left[4\left|\varphi_{11}\right|^{2}+\left(\Delta_{b} \varphi\right)^{2}+6\left\langle\nabla_{b} \varphi, \nabla_{b} \Delta_{b} \varphi\right\rangle+\frac{3}{4} \Delta_{b} \varphi_{t}\right. \\
& -8 W^{-2}\left\langle P W+\bar{P} W, d_{b} W\right\rangle_{L_{\theta}^{*}}+4\left\langle\nabla_{b} \varphi, \nabla_{b}\left|\nabla_{b} \varphi\right|^{2}\right\rangle \\
& \left.+4\left(\Delta_{b} \varphi+\left|\nabla_{b} \varphi\right|^{2}\right)\left|\nabla_{b} \varphi\right|^{2}-2 W\left(6 \Delta_{b} \varphi+5\left|\nabla_{b} \varphi\right|^{2}\right)\right],
\end{aligned}
$$

where we have used the inequalities

$$
\begin{aligned}
& \left|\left(\nabla^{H}\right)^{2} \varphi\right|^{2}=2\left|\varphi_{11}\right|^{2}+\frac{1}{2}\left(\Delta_{b} \varphi\right)^{2}+\frac{1}{2} \varphi_{0}^{2} \geq 2\left|\varphi_{11}\right|^{2}+\frac{1}{2}\left(\Delta_{b} \varphi\right)^{2}, \\
& (2 \operatorname{Ric}-3 \operatorname{Tor})\left(\left(\nabla_{b} \varphi\right)_{C},\left(\nabla_{b} \varphi\right)_{C}\right)=2 W\left|\left(\nabla_{b} \varphi\right)_{C}\right|^{2}=W\left|\nabla_{b} \varphi\right|^{2}
\end{aligned}
$$

and

$$
W^{-1} \Delta_{b} W=\Delta_{b} \varphi+\left|\nabla_{b} \varphi\right|^{2}
$$

Applying the formula

$$
\Delta_{b} \varphi=\frac{1}{4} \varphi_{t}-\left|\nabla_{b} \varphi\right|^{2}-\frac{1}{2}(W-r)=\frac{1}{3 t} F-\frac{4}{3}\left|\nabla_{b} \varphi\right|^{2}+\frac{7}{2}(W-r)
$$


and combining (3.8) and (3.9), we conclude that

$$
\begin{aligned}
& \left(\Delta_{b}-\partial_{t} / 4\right) F \\
\geq & -\frac{1}{4 t} F+t\left[4\left|\varphi_{11}\right|^{2}+\left(\Delta_{b} \varphi\right)^{2}+4\left(\Delta_{b} \varphi+\left|\nabla_{b} \varphi\right|^{2}\right)\left|\nabla_{b} \varphi\right|^{2}\right. \\
& +6\left\langle\nabla_{b} \varphi, \nabla_{b} \Delta_{b} \varphi\right\rangle+4\left\langle\nabla_{b} \varphi, \nabla_{b}\left|\nabla_{b} \varphi\right|^{2}\right\rangle-2\left\langle\nabla_{b} \varphi, \nabla_{b} \varphi_{t}\right\rangle \\
& +\left(\frac{3}{2} r-3 W\right) \Delta_{b} \varphi+2 r\left|\nabla_{b} \varphi\right|^{2}+\frac{21}{4} W(W-r)-\frac{21}{8} r_{t} \\
& \left.-8 W^{-2}\left\langle P W+\bar{P} W, d_{b} W\right\rangle_{L_{\theta}^{*}}\right] \\
= & -\frac{1}{4 t} F+t\left[4\left|\varphi_{11}\right|^{2}+\left(\Delta_{b} \varphi\right)^{2}+4\left(\Delta_{b} \varphi+\left|\nabla_{b} \varphi\right|^{2}\right)\left|\nabla_{b} \varphi\right|^{2}\right. \\
& -\frac{2}{3 t}\left\langle\nabla_{b} \varphi, \nabla_{b} F\right\rangle-\frac{4}{3}\left\langle\nabla_{b} \varphi, \nabla_{b}\left|\nabla_{b} \varphi\right|^{2}\right\rangle+\left(\frac{3}{2} r-3 W\right) \Delta_{b} \varphi \\
& +(2 r-11 W)\left|\nabla_{b} \varphi\right|^{2}+\frac{21}{4} W(W-r)-\frac{21}{8} r_{t} \\
& \left.-8 W^{-2}\left\langle P W+\bar{P} W, d_{b} W\right\rangle_{L_{\theta}^{*}}\right] .
\end{aligned}
$$

Now, it is easy to see that

$$
\left\langle\nabla_{b} \varphi, \nabla_{b}\left|\nabla_{b} \varphi\right|^{2}\right\rangle=4 \operatorname{Re}\left(\varphi_{11} \varphi_{\overline{1}} \varphi_{\overline{1}}\right)+\Delta_{b} \varphi\left|\nabla_{b} \varphi\right|^{2},
$$

thus

$$
\begin{aligned}
-\frac{4}{3}\left\langle\nabla_{b} \varphi, \nabla_{b}\left|\nabla_{b} \varphi\right|^{2}\right\rangle & =-\frac{16}{3} \operatorname{Re}\left(\varphi_{11} \varphi_{\overline{1}} \varphi_{\overline{1}}\right)-\frac{4}{3} \Delta_{b} \varphi\left|\nabla_{b} \varphi\right|^{2} \\
& \geq-4\left|\varphi_{11}\right|^{2}-\frac{16}{9}\left|\varphi_{\overline{1}}\right|^{4}-\frac{4}{3} \Delta_{b} \varphi\left|\nabla_{b} \varphi\right|^{2} \\
& =-4\left|\varphi_{11}\right|^{2}-\frac{4}{9}\left|\nabla_{b} \varphi\right|^{4}-\frac{4}{3} \Delta_{b} \varphi\left|\nabla_{b} \varphi\right|^{2} .
\end{aligned}
$$

Here we used the basic inequality $2 \operatorname{Re}(z w) \leq \epsilon|z|^{2}+\epsilon^{-1}|w|^{2}$ for all $\epsilon>0$. Substituting this inequality into (3.11) and applying the formula (3.10), we finally get

$$
\begin{aligned}
& \left(\Delta_{b}-\frac{\partial}{\partial t}\right) F \\
\geq \quad & -\frac{2}{3}\left\langle\nabla_{b} \varphi, \nabla_{b} F\right\rangle+t\left[\left(\Delta_{b} \varphi\right)^{2}+\frac{8}{3} \Delta_{b} \varphi\left|\nabla_{b} \varphi\right|^{2}+\frac{32}{9}\left|\nabla_{b} \varphi\right|^{4}\right. \\
& +\left(\frac{3}{2} r-3 W\right) \Delta_{b} \varphi+(2 r-11 W)\left|\nabla_{b} \varphi\right|^{2}+\frac{21}{4} W(W-r) \\
& \left.-\frac{21}{8} r_{t}-8 W^{-2}\left\langle P W+\bar{P} W, d_{b} W\right\rangle_{L_{\theta}^{*}}\right]-\frac{1}{4 t} F \\
= & -\frac{2}{3}\left\langle\nabla_{b} \varphi, \nabla_{b} F\right\rangle+\frac{1}{9 t} F\left[F+\left(12 W-\frac{33}{2} r\right) t-\frac{9}{4}\right] \\
& +\frac{16}{9} t\left|\nabla_{b} \varphi\right|^{4}-7 t W\left|\nabla_{b} \varphi\right|^{2}+7 t(W-r)^{2}-\frac{21}{8} t r_{t} \\
& -8 t W^{-2}\left\langle P W+\bar{P} W, d_{b} W\right\rangle_{L_{\theta}^{*}} .
\end{aligned}
$$

This proves the lemma.

In order to deal with the term $\left\langle P W+\bar{P} W, d_{b} W\right\rangle_{L_{\theta}^{*}}$ in Lemma 3.8, we assume the torsion vanishes at the initial time. Then by Lemma 3.3, the torsion $A_{11}$ vanishes 
identically for all time; so does $W_{0}$ due to the Bianchi identity ([L2]):

$$
A_{11, \overline{1} \overline{1}}+A_{\overline{1} \overline{1}, 11}=W_{0} .
$$

This implies $W_{0}$ vanishes identically for all time. It follows that $W_{1 \overline{1}}=W_{\overline{1} 1}$ and $W_{10}=W_{01}$ by the commutation relations. In addition, when the CR structure $J$ is spherical, we have $W_{11}=0$ for all time due to the fact that the Cartan curvature tensor $Q_{11}$ vanishes identically.

Lemma 3.9. Suppose $J$ is spherical and the torsion $A_{11}$ vanishes identically. Then we have

$$
\left\langle P W+\bar{P} W, d_{b} W\right\rangle_{L_{\theta}^{*}}=-W\left|\nabla_{b} W\right|^{2} .
$$

Proof. Using the commutation relations ([L2]) and the Bianchi identity (3.12), we obtain

$$
\begin{aligned}
W_{11 \overline{1}} & =W_{1 \overline{1} 1}+i W_{10}+W W_{1} \\
& =W_{\overline{1} 11}+i W_{01}+W W_{1} \\
& =W_{\overline{1} 11}+W W_{1} .
\end{aligned}
$$

This implies that $W_{\overline{1} 11}=-W W_{1}$. Thus

$$
\begin{aligned}
\left\langle P W+\bar{P} W, d_{b} W\right\rangle_{L_{\theta}^{*}} & =W_{\overline{1} 11} W_{\overline{1}}+W_{1 \overline{1} \overline{1}} W_{1} \\
& =-2 W W_{1} W_{\overline{1}}=-W\left|\nabla_{b} W\right|^{2} .
\end{aligned}
$$

Now we are ready to derive the subgradient estimate for the positive solution of the Tanaka-Webster curvature $W(x, t)$ of (3.1) on $M \times(0, T]$.

Proof of Theorem 1.4. Apply Lemma 3.8 to obtain $\varphi=\log W$, Lemma 3.2. Lemma 3.3. and Lemma 3.9 to get $W^{-2}\left\langle P W+\bar{P} W, d_{b} W\right\rangle_{L_{\theta}^{*}}=-W\left|\nabla_{b} \varphi\right|^{2}$, and Lemma 3.4 to obtain $r_{t} \leq 0$. Combining all of these we have

$$
\begin{aligned}
\left(\Delta_{b}-\partial_{t} / 4\right) F \geq & -\frac{2}{3}\left\langle\nabla_{b} \varphi, \nabla_{b} F\right\rangle+\frac{1}{9 t} F\left[F+\left(12 W-\frac{33}{2} r\right) t-\frac{9}{4}\right] \\
& +\frac{16}{9} t\left|\nabla_{b} \varphi\right|^{4}+t W\left|\nabla_{b} \varphi\right|^{2}+7 t(W-r)^{2}-\frac{21}{8} t r_{t} \\
\geq & -\frac{2}{3}\left\langle\nabla_{b} \varphi, \nabla_{b} F\right\rangle+\frac{1}{9 t} F\left[F+\left(12 W-\frac{33}{2} r\right) t-\frac{9}{4}\right] \\
& +7 t(W-r)^{2} .
\end{aligned}
$$

We claim that $F$ is at most $\left(\frac{33}{2} r-12 W\right) t+\frac{9}{4}$. If not, at the maximum point $\left(x_{0}, t_{0}\right)$ of $F$ on $M \times[0, T]$,

$$
F\left(x_{0}, t_{0}\right)>\left(\frac{33}{2} r_{\left(t_{0}\right)}-12 W\left(x_{0}, t_{0}\right)\right) t_{0}+\frac{9}{4} .
$$

Clearly, $t_{0}>0$, because $F(x, 0)=0$. By the fact that $\left(x_{0}, t_{0}\right)$ is a maximum point of $F$ on $M \times[0, T]$, we have

$$
\Delta_{b} F\left(x_{0}, t_{0}\right) \leq 0, \quad \nabla_{b} F\left(x_{0}, t_{0}\right)=0,
$$

and

$$
F_{t}\left(x_{0}, t_{0}\right) \geq 0
$$


Combining this with (3.13) implies

$$
\begin{aligned}
0 \geq & \frac{1}{9 t_{0}} F\left(x_{0}, t_{0}\right)\left[F\left(x_{0}, t_{0}\right)+\left(12 W\left(x_{0}, t_{0}\right)-\frac{33}{2} r_{\left(t_{0}\right)}\right) t_{0}-\frac{9}{4}\right] \\
& +7 t_{0}\left(W\left(x_{0}, t_{0}\right)-r_{\left(t_{0}\right)}\right)^{2},
\end{aligned}
$$

which is a contradiction. Since we always have $F\left(x_{0}, t_{0}\right) \geq 0$ and if $W\left(x_{0}, t_{0}\right)=$ $r_{\left(t_{0}\right)}$, then $F\left(x_{0}, t_{0}\right)>\frac{9}{4}\left(2 r_{\left(t_{0}\right)} t_{0}+1\right)>0$. Hence we obtain

$$
F \leq\left(\frac{33}{2} r-12 W\right) t+\frac{9}{4}
$$

as desired, which implies

$$
\left|\nabla_{b} \varphi\right|^{2}+\frac{3}{4} \varphi_{t}-12(W-r) \leq\left(\frac{33}{2} r-12 W\right)+\frac{9}{4 t} .
$$

This completes the proof of the theorem.

Proof of Theorem 1.5. Let $\gamma$ be a Legendrian path with $\gamma\left(t_{1}\right)=x_{1}$ and $\gamma\left(t_{2}\right)=x_{2}$. We define $\eta:\left[t_{1}, t_{2}\right] \rightarrow M \times\left[t_{1}, t_{2}\right]$ by

$$
\eta(t)=(\gamma(t), t) .
$$

Clearly $\eta\left(t_{1}\right)=\left(x_{1}, t_{1}\right)$ and $\eta\left(t_{2}\right)=\left(x_{2}, t_{2}\right)$. Letting $\varphi=\log W(x, t)$ and integrating $\frac{d}{d t} \varphi$ along $\eta$, we get

$$
\varphi\left(x_{2}, t_{2}\right)-\varphi\left(x_{1}, t_{1}\right)=\int_{t_{1}}^{t_{2}} \frac{d}{d t} \varphi d t=\int_{t_{1}}^{t_{2}}\left(\left\langle\dot{\gamma}, \nabla_{b} \varphi\right\rangle+\varphi_{t}\right) d t
$$

Applying Theorem 1.4 to $\varphi_{t}$ yields

$$
\begin{aligned}
\varphi\left(x_{2}, t_{2}\right)-\varphi\left(x_{1}, t_{1}\right) & \leq \int_{t_{1}}^{t_{2}}\left(|\dot{\gamma}|\left|\nabla_{b} \varphi\right|+\varphi_{t}\right) d t \\
& \leq \int_{t_{1}}^{t_{2}}\left(\frac{3}{16}|\dot{\gamma}|^{2}+6 r+\frac{3}{t}\right) d t \\
& =\int_{t_{1}}^{t_{2}} \frac{3}{16}|\dot{\gamma}|^{2} d t+6 \int_{t_{1}}^{t_{2}} r d t+3 \log \left(\frac{t_{2}}{t_{1}}\right) .
\end{aligned}
$$

The theorem follows by taking exponentials of the above inequality.

\section{Compactness of PSEUdohermitian structures WITH INTEGRAL BOUNDS ON THE CURVATURE}

Let $(M, J, \theta)$ be a closed 3 -dimensional pseudohermitian manifold. Let $\left\{\theta_{k}=\right.$ $\left.u_{k}^{2} \theta, u_{k}>0\right\}$ be a sequence of contact forms which satisfy

$$
\begin{gathered}
\operatorname{Vol}\left(h_{k}\right)=\int u_{k}^{4} d \mu \leqslant V_{0}, \\
\int\left|W_{k}\right|^{p} d \mu_{k} \leqslant \beta^{p}, \\
\int\left|\tau_{k}^{1}\right|_{k}^{p} d \mu_{k}=\int\left|A_{\overline{1} \overline{1}}(k)\right|^{p} d \mu_{k} \leqslant \beta^{p}, \\
\int\left|A_{\overline{1} \overline{1}, 1}(k)\right|^{p} d \mu_{k} \leqslant \beta^{p}, \\
\int\left|W_{k, 0}\right|^{2} d \mu_{k} \leqslant \beta^{2}
\end{gathered}
$$


for some constants $V_{0}, \beta$ and $p>2$. Here $W_{k}$ and $\tau_{k}^{1}$ denote the Tanaka-Webster curvature and the pseudohermitian torsion with respect to $\left(J, \theta_{k}=u_{k}^{2} \theta\right), A_{\overline{1} \overline{1}, 1}(k)$ is the covariant derivative of $A_{\overline{1} \overline{1}}(k)$ and $W_{k, 0}=T_{k} W_{k}$. Also, $d \mu_{k}=u_{k}^{4} d \mu$ is the volume form with respect to the adapted metric $h_{k}=d \theta_{k}(\cdot, J \cdot)+\theta_{k}^{2}$ and $d \mu$ is the volume form with respect to $h=d \theta(\cdot, J \cdot)+\theta^{2}$.

Before stating the main theorem, we first define a somewhat technical condition on $\left\{u_{k}\right\}$, say condition $(*)$, as follows:

Definition 4.1. We say that $\left\{u_{k}\right\}$ satisfies $(*)$ if there is a point $x \in M$, and constants $\rho, \varepsilon, C>0$ such that

$$
\int_{B(x, \rho)} u_{k}^{-\varepsilon} d \mu \leqslant C
$$

where $B(x, \rho)$ denotes the ball of radius $\rho$ centered at $x$ with respect to a natural distance function.

The condition $(*)$ was imposed in the works of Gursky $([\mathrm{Gu}])$, Chang $([\mathrm{Ch}])$ and Chang-Wu $([\mathrm{CW}])$. In the rest of this section, we consider a contact class $[\theta]$ with $\theta_{k}=u_{k}^{2} \theta$, for $u_{k}>0$. Note that

$$
\left(2 \Delta_{b}+W\right) u_{k}=W_{k} u_{k}^{3}
$$

It follows (Chi]) that

Lemma 4.2. Suppose that $\left\{u_{k}\right\}$ satisfies (4.1), (4.2), and (*). Then there are constants $\delta_{0}, C_{0}^{\prime}>0$ such that

$$
\int_{M} u_{k}^{-\delta_{0}} d \mu \leqslant C_{0}^{\prime}
$$

As a consequence, there is a constant $C_{0}\left(V_{0}, \beta\right)>0$ such that

$$
u_{k} \geqslant C_{0} \text {. }
$$

Proposition 4.3. Suppose that $\left\{u_{k}\right\}$ satisfies (4.1), (4.2), (4.3), (4.4), (4.5). In addition, if $\left\{u_{k}\right\}$ has a uniform lower bound, then there are constants $C_{1}, C_{2}$, which depend on $V_{0}, \beta$ and $p$, such that

$$
C_{1}^{-1} \leqslant u_{k} \leqslant C_{1}
$$

and

$$
\left\|u_{k}\right\|_{S_{2}^{p}(M)} \leqslant C_{2} \text {. }
$$

As a consequence of Lemma 4.2 and Proposition 4.3, one has

Corollary 4.4. Suppose that $\left\{u_{k}\right\}$ satisfies (4.1), (4.2), (4.3), (4.4), (4.5) and (*). Then $\left\{u_{k}\right\}$ satisfies the conclusions (4.9) and (4.10) of the previous proposition.

Now the main step is to establish the condition $(*)$ for $\left\{u_{k}\right\}$. To this end, we have ([Chi1 $]$ ).

Lemma 4.5. Let $(M, J, \theta)$ be a closed 3-dimensional pseudohermitian manifold with a negative $C R$ Yamabe constant and where $\left\{u_{k}\right\}$ satisfies (4.1), (4.2), (4.3), (4.4), (4.5). Then $\left\{u_{k}\right\}$ satisfies $(*)$. 
Lemma 4.6. Let $(M, J, \theta)$ be a closed 3-dimensional pseudohermitian manifold which is not $C R$ spherical and where $\left\{u_{k}\right\}$ satisfies (4.1), (4.2), (4.3), (4.4), (4.5). Then $\left\{u_{k}\right\}$ satisfies $(*)$, provided that there also exists an $L^{p}$ bound on the Cartan tensor,

$$
\int\left|Q_{J}\left(\theta_{k}\right)\right|_{k}^{p} d \mu_{k} \leqslant \beta^{p}, \quad p>2 .
$$

In this section, we shall extend Lemma 4.6 to a closed spherical 3-dimensional pseudohermitian manifold as well. Now let $D=\left\{\zeta \in \mathbb{C}^{2}:|\zeta|<1\right\}$ be the unit ball and $\Omega=\left\{(z, w) \in \mathbb{C}^{2}: \operatorname{Im} w>|z|^{2}\right\}$ be the Siegel upper half space. The Cayley transform : $D \rightarrow \Omega$ is a biholomorphism given by

$$
w=i\left(\frac{1-\zeta_{2}}{1+\zeta_{2}}\right), \quad z=\frac{\zeta_{1}}{1+\zeta_{2}} .
$$

When restricted to the boundary, this transformation gives a $\mathrm{CR}$ equivalence between $\mathrm{S}^{3}=\partial D$ minus the south pole and $\partial \Omega$. The Heisenberg group $H^{1}$ is identified with $\partial \Omega$ by $(z, t) \rightarrow\left(z, t+i|z|^{2}\right)$. Define the mapping $F: \mathrm{S}^{3} \rightarrow H^{1}$ given by (4.12), followed by this identification $H^{1}=\partial \Omega$. We write

$$
\theta_{1}=i(\bar{\partial}-\partial)|\zeta|^{2}=i\left(\sum_{j=1}^{2} \zeta_{j} d \overline{\zeta_{j}}-\overline{\zeta_{j}} d \zeta_{j}\right)
$$

as the standard contact form on the sphere. Then

$$
\theta_{1}=F^{*}\left(\frac{4}{|i+w|^{2}} \theta_{0}\right)=F^{*}\left(\frac{4}{\left.\left.|i+t+i| z\right|^{2}\right|^{2}} \theta_{0}\right)
$$

and

$$
\left(F^{-1} d_{\delta} F\right)^{*} \theta_{1}=f_{\delta}^{2} \theta_{1}
$$

with

$$
f_{\delta}=\frac{2 \delta}{\left|\left(1+\zeta_{2}\right)+\delta^{2}\left(1-\zeta_{2}\right)\right|} .
$$

Here $\theta_{0}=d t+i(z d \bar{z}-\bar{z} d z)$ is the standard contact form on $H^{1}$ and $d_{\delta} x=\left(\delta z, \delta^{2} t\right)$ is the dilation.

Now, following the lines of M. J. Gursky's result ([Gu, Proposition 4.4.]), we show that

Theorem 4.7. Suppose that $(M, J, \theta)$ is a closed 3-dimensional pseudohermitian manifold which is $C R$ equivalent to $\left(\mathbf{S}^{3}, \widehat{J}\right)$ and that $\left\{u_{k}\right\}$ satisfies conditions (4.1) and (4.2). Then if $\left\{u_{k}\right\}$ does not satisfy $(*)$, there is the CR automorphism $\left\{T_{k}\right\}$ such that, if $T_{k}^{*} \theta_{k}=v_{k}^{2} \theta_{0}$, then $\left\{v_{k}\right\}$ satisfies $(*)$.

Proof. The following idea of renormalizing is due to K. Uhlenbeck. Let us assume that $(M, J)=\left(\mathrm{S}^{3}, \widehat{J}\right)$. The more general case of $\mathrm{CR}$ equivalence follows in an obvious way.

If $\left\{u_{k}\right\}$ does not satisfy $(*)$, then $\max u_{k} \rightarrow \infty$ as $k \rightarrow \infty$. After composing with a rotation which is a CR automorphism, we can assume that the maximum of $u_{k}$ is achieved at the point $\left(\zeta_{1}, \zeta_{2}\right)=(0,0,1,0) \in S^{3} \subset \mathbb{C}^{2}$. Let $\varphi_{\delta}=F^{-1} d_{\delta} F$. Then $\varphi_{\delta}$ is a CR automorphism and $(0,0,1,0)$ is a fixed point of $\varphi_{\delta}$. Moreover,

$$
\varphi_{\delta}^{*} \theta=f_{\delta}^{2} \theta
$$


where

Put $\varphi_{\delta}^{*} \theta_{k}=v_{k, \delta}^{2} \theta ;$ then

$$
f_{\delta}=\frac{2 \delta}{\left|\left(1+\zeta_{2}\right)+\delta^{2}\left(1-\zeta_{2}\right)\right|} .
$$

$$
v_{k, \delta}=f_{\delta}\left(\zeta_{1}, \zeta_{2}\right)\left(u_{k} \circ \varphi_{\delta}\right)\left(\zeta_{1}, \zeta_{2}\right) .
$$

For each $k$, choose $\delta_{k}$ so that $v_{k, \delta_{k}}(0,0,1,0)=v_{k}(0,0,1,0)=1\left(\delta_{k}=\frac{1}{u_{k}(0,0,1,0)}\right)$.

Then

$$
\begin{aligned}
v_{k}\left(\zeta_{1}, \zeta_{2}\right) & =f_{\delta_{k}}\left(\zeta_{1}, \zeta_{2}\right)\left(u_{k} \circ \varphi_{\delta_{k}}\right)\left(\zeta_{1}, \zeta_{2}\right) \\
& \leq f_{\delta_{k}}\left(\zeta_{1}, \zeta_{2}\right) u_{k}(0,0,1,0) \\
& =\frac{2}{\left|\left(1+\zeta_{2}\right)+\delta_{k}^{2}\left(1-\zeta_{2}\right)\right|} \\
& \leq C
\end{aligned}
$$

if $\operatorname{Im} \zeta_{2}<-\frac{1}{2}$.

Now let $\Omega=\left\{\left(\zeta_{1}, \zeta_{2}\right) \in \mathrm{S}^{3} \mid \operatorname{Im} \zeta_{2}<-\frac{1}{2}\right\}$. By using (4.6) and (4.2), it follows from the Harnack inequality ([JL1] and [Tru]) that

$$
1 \leq \sup _{\Omega} v_{k} \leq C\left(V_{0}, \beta\right) \inf _{\Omega} v_{k}
$$

This gives a uniformly lower bound of $\left\{v_{k}\right\}$ on $\Omega$ and then implies that $\left\{v_{k}\right\}$ satisfies $(*)$.

Due to Proposition 5.1 of $\mathrm{Gu}$, we expect that

Conjecture 4.8. Suppose $(M, J, \theta)$ is a closed spherical pseudohermitian 3-manifold with $\lambda(M)>0$. If $\left\{u_{k}\right\}$ does not satisfy condition $(*)$, then $(M, J)$ is $C R$ equivalent to $\left(\mathbf{S}^{3}, \widehat{J}\right)$.

\section{The ASYMPtotic CONVERGENCE OF SOLUTiONS OF THE CR YAMABE FLOW}

In this section, under the CR Yamabe flow we first derive the uniformly $L^{p}$ bound estimate of $W$ from the subgradient estimate in Theorem 1.4. By combining the compactness theorem of a sequence of contact forms as in the previous section, we are able to get the uniform $S^{2, p}$-estimates for any fixed $p>2$. Now the standard subelliptic theory and bootstrapping then yield the uniform smooth estimates for $\lambda$.

Lemma 5.1. Under the flow (1.1) we have

$$
\int_{M} W^{2} d \mu \leq C_{0}\left(W_{(0)}, \theta_{(0)}\right)
$$

for $0 \leq t \leq T \leq \infty$.

Proof. From (3.1), we compute

$$
\begin{aligned}
-\frac{1}{2} \frac{d}{d t} \int_{M} W^{2} d \mu & =-2 \int_{M} W^{2}(r-W) d \mu-\int_{M} W\left[4 \Delta_{b} W+2 W(W-r)\right] d \mu \\
& =-4 \int_{M} W \Delta_{b} W d \mu=4 \int_{M}\left|\nabla_{b} W\right|^{2} d \mu .
\end{aligned}
$$

Thus

$$
\frac{d}{d t} \int_{M} W^{2} d \mu \leq 0 .
$$


Then we use the subgradient estimate in Theorem 1.4 to get a uniform $L^{p}$-bound of $W$ for any $p>2$ after a short time $\tau>0$.

Lemma 5.2. Under the flow (1.1), after a short time $\tau>0$, for any $p>2$, there exists a constant $C_{p}=C\left(p, W_{(0)}, \theta_{(0)}, \tau\right)$ such that

$$
\int_{M} W^{p} d \mu \leq C_{p}
$$

for $0<\tau \leq t \leq T \leq \infty$.

Proof. From Theorem 1.4, we have

$$
\frac{W_{t}}{W} \leq \frac{4}{3} \frac{\left|\nabla_{b} W\right|^{2}}{W^{2}}+\frac{W_{t}}{W} \leq 6 r+\frac{3}{t} \leq 6 r_{(\tau)}+\frac{3}{\tau} \text { for } t \geq \tau .
$$

This implies that

$$
W_{t} \leq C\left(r_{(\tau)}, \tau\right) W
$$

for all $t \geq \tau$. Then, for any $p>2$, we compute

$$
\begin{aligned}
\frac{d}{d t} \int_{M} W^{p} d \mu & =4 \int_{M} W^{p}(r-W) d \mu+p \int_{M} W^{p-1} W_{t} d \mu \\
& \leq-4 \int_{M} W^{p+1} d \mu+C\left(r_{(\tau)}, \tau\right) p \int_{M} W^{p} d \mu
\end{aligned}
$$

and by using the inequality

$$
\int_{M} W^{p} d \mu \leq \varepsilon \int_{M} W^{p+1} d \mu+C(p, \varepsilon) \int_{M} W^{2} d \mu, \text { for any } \varepsilon>0 .
$$

We deduce for some small $\varepsilon>0$

$$
\frac{d}{d t} \int_{M} W^{p} d \mu+3 \int_{M} W^{p+1} d \mu \leq C_{p}^{\prime}
$$

and then

$$
\frac{d}{d t} \int_{M} W^{p} d \mu \leq C_{p}-\int_{M} W^{p} d \mu+C \int_{M} W^{p-1} d \mu .
$$

In particular,

It follows that

$$
\frac{d}{d t} \int_{M} W^{3} d \mu \leq C-\int_{M} W^{3} d \mu .
$$

and for any arbitrary fixed $p$,

$$
\int_{M} W^{3} d \mu \leq C_{3}
$$

$$
\int_{M} W^{p} d \mu \leq C_{p}
$$

It follows from Lemma 3.3. Lemma 5.2 (3.12) and Theorem 4.7 that all assumptions of Corollary 4.4 are satisfied. Then

Corollary 5.3. Under the Yamabe flow (1.1), there are constants $C_{1}$ and $C_{2}$ depending on $C_{p}, \theta_{(0)}$ such that

$$
-C_{1} \leq \lambda \leq C_{1} \text { and }\|\lambda\|_{W_{2, p}} \leq C_{2}
$$

hold up to the CR automorphism for all time. This leads to a Hölder continuity estimate for $\lambda$ for all time. 
Proof of Theorem [1.6. From Corollary 5.3 the standard subelliptic theory and bootstrapping yield the uniform smooth estimates for $\lambda$ ([JL1], [Y], [SS]). Now by L. Simon's general results $\underline{\underline{S}}$, the solutions of the Yamabe flow (1.1) converge smoothly to a unique limit contact form at $t \rightarrow \infty$. On the other hand, since

$$
-\frac{d}{d t} r=2 \int_{M}(W-r)^{2} d \mu / \int_{M} d \mu
$$

and the volume of $M$ is preserved along the flow,

$$
\int_{0}^{\infty} \int_{M}(W-r)^{2} d \mu d t<\infty
$$

It follows that the limit contact form has constant Webster scalar curvature. This completes the proof of Theorem 1.6 .

\section{ACKNOWLEDGMENTS}

The first author would like to express his thanks to Professor S.-T. Yau for his inspirations on Li-Yau inequality and for his support to make this work possible. The authors would also like to express their thanks to the referee for valuable comments.

\section{REFERENCES}

[A] T. Aubin, Some Nonlinear Problems in Riemannian Geometry, Springer Monographs in Mathematics, Springer-Verlag, Berlin, 1998. MR 1636569 (99i:58001)

[C] B. Chow, The Yamabe Flow on Locally Conformally Flat Manifolds with Positive Ricci Curvature, Comm. Pure Appl. 45(1992), 1003-1014. MR1168117 (93d:53045)

[CC] S.-C. Chang and J.-H. Cheng, The Harnack Estimate for the Yamabe Flow on CR Manifolds of Dimension 3, AGAG, 21 (2002), 111-121. MR.1894940 (2002m:53055)

[CCC] S.-C. Chang, J.-H. Cheng and H.-L. Chiu, The Fourth-order Q-curvature Flow on a CR 3Manifold, Indiana Univ. Math. J., 56, No. 4 (2007), 1793-1826. MR2354700|(2009a:53111)

[CCh] S.-C. Chang and H.-L. Chiu, On the Estimate of First Eigenvalue of a Sublaplacian on a Pseudo-Hermitian 3-Manifold, Pacific Journal of Mathematics, 232, No. 2 (2007), 269-282. MR.2366354 (2008m:58065)

[CCW] S.-C. Chang, H.-L. Chiu and C.-T. Wu, Subgradient Estimate, Eigenvalue Estimates and Li-Yau Inequality in Pseudohermitian 3-Manifolds, preprint.

[Ch] S.-C. Chang, The 2-Dimensional Calabi Flow, Nagoya Math. J., 181 (2006), 63-73. MR2210710 (2006j:53095)

[Chi1] H.-L. Chiu, Compactness of Pseudohermitian Structures with Integral Bounds on Curvature, Math. Ann., 334 (2006), 111-142. MR.2208951 (2007d:32028)

[Chi2] H.-L. Chiu, The Sharp Lower Bound for the First Positive Eigenvalue of the Sublaplacian on a Pseudohermitian 3-Manifold, Annals of Global Analysis and Geometry, 30 (2006), 81-96. MR 2249615 (2007j:58034)

[Cho] W.-L. Chow, Uber System Von Lineaaren Partiellen Differentialgleichungen erster Orduung, Math. Ann., 117 (1939), 98-105. MR0001880(1:313d)

[CW] S.-C. Chang and C.-T. Wu, On the Existence of Extremal Metrics on Complete Noncompact 3-Manifolds, Indiana Univ. Math. J., 53 (2004), 243-268. MR2048993 (2005a:53057)

[CY] H.-D. Cao and S.-T. Yau, Gradient Estimates, Harnack Inequalities and Estimates for Heat Kernels of the Sum of Squares of Vector Fields, Math. Z., 211 (1992), 485-504. MR.1190224 (94h:58155)

[GY] N. Gamara and Y. Yacoub, The CR Yamabe conjecture-the case $n=1$, J. Eur. Math. Soc. 3 (2001), 105-137. MR1831872 (2003d:32040a)

$[\mathrm{H}] \quad$ R. S. Hamilton, The Ricci Flow on Surfaces, Mathematics and General Relativity (Santa Cruz, CA, 1986), 237-262, Contemp. Math., 71, Amer. Math. Soc., Providence, RI, 1998. MR954419 (89i:53029)

[JL1] D. Jerison and J. M. Lee, The Yamabe Problem on $C R$ manifolds, J. Diff. Geom., 25 (1987), 167-197. MR880182(88i:58162) 
[JL2] Extremals for the Sobolev Inequality on the Heisenberg Group and the $C R$ Yamabe Problem, J. Amer. Math. Soc., 1 (1988), 1-13.

[JL3] - Intrinsic $C R$ Normal Coordinates and the $C R$ Yamabe Problem, J. Diff. Geom., 29 (1989), 303-343.

[L1] J. M. Lee, The Fefferman Metric and Pseudohermitian Invariants, Trans. Amer. Math. Soc., 296 (1986), 411-429. MR837820 (87j:32063)

[L2] - Pseudo-Einstein Structures on $C R$ Manifolds, Am. J. Math., 110 (1988), 157-178. MR0926742 (89f:32034)

[LY] P. Li and S.-T. Yau, On the Parabolic Kernel of the Schrödinger Operator, Acta Math., 156 (1985), 153-201. MR834612 (87f:58156)

[Fo] G. B. Folland, Subelliptic Estimates and Function Spaces on Nilpotent Lie Groups, Ark. Mat. 13 (1975), 161-207. MR0494315 (58:13215)

[FS] G. B. Folland and E. M. Stein, Estimates for the $\bar{\partial}_{b}$ Complex and Analysis on the Heisenberg Group, Comm. Pure Appl. Math., 27 (1974), 429-522. MR0367477 (51:3719)

[Gu] M.J. Gursky, Compactness of Conformal Metrics with Integral Bounds on Curvature, Duke Math. J. Vol. 72, No. 2 (1993), 339-367. MR.1248676 (94m:53054)

[S] L. Simon, Asymptotics for a Class of Nonlinear Evolution Equations, with Applications to Geometric Problems, Ann. of Math., 118 (1983), 525-571. MR727703 (85b:58121)

[Sch] R. Schoen, Conformal Deformation of a Riemannian Metric to a Constant Scalar Curvature, J. Diff. Geom., 20 (1984), 479-495. MR788292 (86i:58137)

[SS] H. Schwetlick and M. Struwe, Convergence of the Yamabe Flow for Large Energies, J. Reine Angew. Math., 562 (2003), 59-100. MR2011332 (2004h:53097)

[SY] R. Schoen and S.-T. Yau, Conformally Flat, Kleinian Groups and Scalar Curvature, Invent. Math. 92 (1988), 47-71. MR931204 (89c:58139)

[T] N. Tanaka, A Differential Geometric Study on Strongly Pseudo-Convex Manifolds, 1975, Kinokuniya Co. Ltd., Tokyo. MR0399517 (53:3361)

[Tru] N. Trudinger, Remarks Concerning the Conformal Deformation of Riemannian Structures on Compact Manifolds, Ann. Scuola Norm. Sup. Pisa CI. Sci. (4), 22 (1968), 265-274. MR0240748 (39:2093)

[W] S. M. Webster, Pseudohermitian Structures on a Real Hypersurface, J. Diff. Geom., 13 (1978), 25-41. MR.520599 (80e:32015)

[Y] R. Ye, Global Existence and Convergence of Yamabe Flow, J. Diff. Geom., 39 (1994), 35-50. MR.1258912 (95d:53044)

Department of Mathematics, National Taiwan University, Taipei 10617, Taiwan, RePUBLIC OF CHINA

E-mail address: scchang@math.ntu.edu.tw

Department of Mathematics, National Central University, Chung-Li 32054, Taiwan, Republic of China

E-mail address: hlchiu@math.ncu.edu.tw

Department of Applied Mathematics, National PingTung University of Education, PingTung 90003, Taiwan, Republic of China

E-mail address: ctwu@mail.npue.edu.tw 